\title{
Anisotropic WM conductivity reconstruction based on diffusion tensor magnetic resonance imaging: a simulation study*
}

\author{
Dandan Yan ${ }^{1}$, Wenlong $\mathrm{Xu}^{1}$, Jing $\mathrm{Li}^{2}$ \\ ${ }^{1}$ College of Information Engineering, China JiLiang University, Hangzhou, China; \\ ${ }^{2}$ Center for THz Research, China Jiliang University, Hangzhou, China. \\ Email: dandanyan@cjlu.edu.cn
}

Received 18 May 2010; revised 8 June 2010; accepted 11 June 2010.

\begin{abstract}
The present study aims to estimate the in vivo anisotropic conductivities of the White Matter (WM) tissues by means of Magnetic Resonance Electrical Impedance Tomography (MREIT) technique. The realistic anisotropic volume conductor model with different conductivity properties (scalp, skull, CSF, gray matter and WM) is constructed based on the Diffusion Tensor Magnetic Resonance Imaging (DT-MRI) from a healthy human subject. The Radius Basic Function (RBF)-MREIT algorithm of using only one magnetic flux density component was applied to evaluate the eigenvalues of the anisotropic WM with target values set according to the DT-MRI data based on the Wolter's model, which is more physiologically reliable. The numerical simulations study performed on the five-layer realistic human head model showed that the conductivity reconstruction method had higher accuracy and better robustness against noise. The pilot research was used to judge the feasibility, meaningfulness and reliability of the MREIT applied on the electrical impedance tomography of the complicated human head tissues including anisotropic characteristics.
\end{abstract}

Keywords: Magnetic Resonance Electrical Impedance Tomography; Radius Basic Function Neural Network; Diffusion Tensor Magnetic Resonance Imaging;

Anisotropic Conductivity; WM

\section{INTRODUCTION}

Knowledge of the electrical conductivity distribution in human body is important to many biomedical applications [1]. Brain disease and brain function activities always accompany with changing conductivities of human

*This work was supported by grants from the Zhejiang Provincial Natural Science Foundation of China (No. Y1080215 and No. Y2090966). head tissues. In the electroencephalography (EEG) or magnetoencephalography (MEG) based source localization or imaging, the conductivity distribution is often assumed to be isotropic and piece-wise homogeneous. However, this assumption is not entirely accurate since the conductivity is highly anisotropic within the WM [1]. The more accurate conductivity distribution of the WM volume is needed for accurate source localization from EEG/MEG.

The cerebral WM is considered as glia and axons bathing in the interstitial fluid. WM shows obvious anisotropy due to its much complicated nerve fiber distribution. Some methods have been reported to get the anisotropic WM conductivity [2,3], which are based on Basser's [4] theory to infer the electrical conductivity tensor from the water self-diffusion tensor measured by diffusion tensor magnetic resonance imaging (DT-MRI) Self-consistent differential effective medium approach (EMA) $[2,5]$ was used to deduce the anisotropic conductivity of the WM tissue: $\sigma \mathrm{WM}=\mathrm{kdW}\left(\mathrm{k}=0.736 \mathrm{~S} \cdot \mathrm{s} / \mathrm{mm}^{3}\right)$ where $\mathrm{dW}$ denoted the eigenvalues of the water diffusion tensor and $\sigma \mathrm{WM}$ was the eigenvalue of the WM conductivity. Wolters [6] has done some study to infer the eigenvalues of the WM considering the nerve fiber structure using the volume constraint and Wang's constraint Wang [7] proposed a new algorithm to derive the anisotropic conductivity of the cerebral WM from the diffusion tensor magnetic resonance imaging data.

The lack of techniques for robust measurement of the electrical conductivity tensor in vivo has discouraged the inclusion of anisotropic conductivity information in the electromagnetic source imaging forward model [8]. Tuch's and other methods all fell back on to deduce the anisotropic WM model directly, which were faced with the difficulties to validate. The research on the electrical characteristic of the head tissues is performed through two ways. In vivo and in vitro measurements of the con- 
ductivities, especially the brain and skull tissues have been published in some literatures [8,9]. Noninvasive MREIT imaging modality has been developed to reconstruct high-resolution conductivity distribution images for the biological tissues.

In this new imaging modality, the traditional Electrical Impedance Tomography (EIT) is combined with magnetic resonance-current density imaging (MR-CDI) technique to solve the well-known ill-posedness of the image reconstruction problem in traditional EIT due to the less effectiveness caused by the low skull conductivity. In MREIT, currents are injected into the subject through pairs of surface electrodes. A Magnetic Resonance Imaging (MRI) scanner is used to measure the induced magnetic flux density inside the subject and the current density distribution can be calculated according to the Ampere's law. The conductivity distribution image can be reconstructed based on the relationship between the conductivity and the measured magnetic flux density combined with the current density. MREIT reconstruction algorithms fall into two categories [10]: those utilizing internal current density [10-14] and those making use of measured magnetic flux density [15-22]. Considering the rotation problem of the object in the MRI system, the latter has the advantage of avoiding the object rotation over the former. Recently, the algorithm based on only one component of the magnetic flux density gains more attention. Several MREIT algorithms have been proposed, which utilize only one component of magnetic flux density, such as the harmonic $B_{z}$ algorithm [14,23], the gradient $B_{z}$ decomposition algorithm [19], the algebraic reconstruction algorithm [18] and an anisotropic conductivity reconstruction algorithm [24].

For the head tissue conductivity, the relatively novel and concise Radial Basis Function (RBF) and Response Surface Methodology (RSM) MREIT algorithms [20,21] have been proposed to focus on the piece-wise homogeneous head tissue conductivity reconstruction. Therefore, RBF-MREIT approach was extended to realize the estimation of anisotropic WM conductivity distribution of the head tissues in this paper. In the present study, a computer simulation study was performed on a fivelayer (scalp, skull, CSF, gray matter and WM) realistic geometry head finite element method (FEM) model. The results showed that the only one component magnetic flux density used could get the same or even better results and confirmed the potential application of the MREIT technology on more complex conductivity distribution reconstruction of human body as well as clinic experiment. The MREIT method could offer a means to the more sophisticated conductivity model including anisotropic tissue.

\section{METHODS}

For the present approach, some assumptions should be given. For the realistic head model, the isotropic conductivities of the scalp, skull, gray matter and CSF were set according to the in-vivo measured value of the literatures $[8,9,25]$. Conductivity tensor of the WM was obtained from the DT-MRI data measurements acquired from a healthy human subject and shared the same eigenvectors with the water diffusion tensors based on Basser's hypothesis [4]. In this simulation study, the target conductivity distribution was set based on the measured tensor data of the WM with the eigenvalue set according to Wolters' [6]. The conductivity tensor map derived from the diffusion tensor image provided the anisotropic conductivity values for each element. The RBF-MREIT [20] algorithm was used to perform the computer simulations on a realistic head model. In the algorithm realization, the current density data and magnetic flux density data were used to estimate the anisotropic conductivity, respectively.

A five-layer (scalp, skull, CSF, gray matter and WM) realistic FEM head model was constructed based on a three-layer FEM head model [26] and T1-MRI data (see Figure 1). FEM was used to solve the forward problem of the MREIT, and then the anisotropic conductivity of the WM was reconstructed by the extended RBF-MREIT algorithm.

\subsection{Realistic FEM Head Model}

An appropriate and sophisticated head model is essential to localize equivalent sources of bioelectric activity of the human brain from the electroencephalogram (EEG). A realistic head model can describe the shape of tissues more accurately.

We first reconstruct a 3D solid model from BEM model and then generate the FEM mesh on the constructed

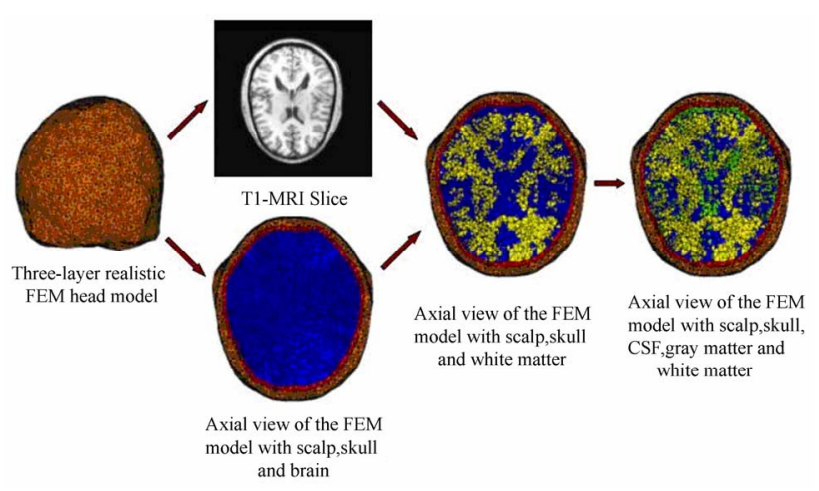

Figure 1. Head model with yellow represents the WM, dark blue the gray matter, green the CSF, red the skull, and brown the skin. 
3D model. The tissue interfaces are described by many surface elements in the BEM model thus the 3D solid model of tissue can be built simply by joining the surface elements into a closed surface. Once given the 3D solid model, the 3D FEM model can be obtained easily by dividing the solid model of the tissue. A CAD software Rhino and a FEM software ANSYS are used.

Based on the T1-weighted MRI data, a realistic fivelayer head model was constructed by FEM and boundary element method (BEM) with 351386 quadratic tetrahedral elements and 485767 nodes. ANSYS 10.0 was used in the finite element modeling and the forward problem calculation based on the EFM realistic head model.

The diffusion distribution of the conductivity could be denoted by a $3 \times 3$ symmetric positive definite ma$\operatorname{trix}: \sigma=\left[\begin{array}{lll}\sigma_{11} & \sigma_{12} & \sigma_{13} \\ \sigma_{21} & \sigma_{22} & \sigma_{23} \\ \sigma_{31} & \sigma_{32} & \sigma_{33}\end{array}\right]$. When $\sigma_{11}=\sigma_{22}=\sigma_{33}$ were constants and other components were zero, the matrix could be rendered as isotropic diffusion conductivity for the grey matter and CSF tissues. For the anisotropic WM, the diffusion distribution of the conductivities in each element (voxel) of the FEM model were written as $\sigma=$ $S \Lambda_{W M} S^{T}$, where $S=\left[\begin{array}{lll}S_{1} & S_{2} & S_{3}\end{array}\right]$ denoted the three unit length orthogonal eigenvectors of the measured diffusion tensor at the center of a WM finite element and $\Lambda_{W M}=$ $\left[\begin{array}{ccc}\lambda_{\text {long }} & 0 & 0 \\ 0 & \lambda_{\text {ltrans }} & 0 \\ 0 & 0 & \lambda_{\text {ltrans }}\end{array}\right]$ was the eigenvalue. $\lambda_{\text {long }}$ and $\lambda_{\text {trans }}$ were the eigenvalue parallel (longitudinal) and perpendicular (trans-verse) to the fiber directions, respectively. The target conductivity distributions of the scalp, skull, CSF, gray matter and WM were set according to the measured data of corresponding tissues [9,25]. The WM was set with anisotropic conductivity distribution based on some existing measured data $[2,6]$ and its anisotropic characteristics.

\subsection{Conductivity Reconstruction}

MREIT imaging modality has been developed to reconstruct high-resolution conductivity distribution images for the biological issues. In this new imaging modality, currents are injected into the subject through pairs of surface electrodes. A Magnetic Resonance Imaging (MRI) scanner is used to measure the induced magnetic flux density $\boldsymbol{B}$ inside the subject and the current density distribution $\boldsymbol{J}$ can be calculated according to the Ampere's law. The conductivity distribution images can be reconstructed based on the relationship between the conductivity and the measured magnetic flux density combined with the current density. MREIT is used to image the conductivities of the human tissues, especially in the human head. The advantages lie in that the magnetic signals tend to penetrate into the inner brain through the low conductivity skull. MREIT reconstruction algorithms mainly fall into two categories: those utilizing internal current density $\boldsymbol{J}$ and those making use of only one component of measured magnetic flux density $\boldsymbol{B}$. Due to the fact that only one component of the magnetic flux density which parallels to the direction of the main magnetic field of the MRI scanner can be measured once, the rotation of the object is required, which is impractical for MRI scanner. Considering the rotation problem, the latter has the advantages over the former of avoiding the object rotation dilemma. MREIT algorithms that are based on current density require knowledge of the magnetic flux density vector $\boldsymbol{B}=\left[\boldsymbol{B}_{x}\right.$, $\left.\boldsymbol{B}_{y}, \boldsymbol{B}_{z}\right]$.

MREIT reconstruction consists of the forward problem and the inverse problem. With given conductivity and boundary condition, the calculation of peripheral voltage values, current density distribution and/or magnetic flux density is referred to as the forward problem of MREIT. The inverse problem deals with the reconstruction of conductivity using the measured magnetic flux density and computed current density information.

\subsubsection{Forward Problem}

Under the quasi-static conditions, the relation between the conductivity and the electrical potential $U(r)$ induced by the injected current is given by Poisson's equation together with the Neumann boundary conditions as:

$$
\begin{aligned}
& \nabla \cdot[\sigma(r) \nabla U(r)]=0, \quad r \in \Omega \\
& \sigma \frac{\partial U}{\partial n}= \begin{cases}J_{i n j} & \text { on positive current electrode } \\
-J_{i n j} & \text { on negative current electrode } \\
0 & \text { elsewhere }\end{cases}
\end{aligned}
$$

where $\sigma(r)$ is the electrical conductivity, $\Omega$, the imaging subject space, $J_{i n j}$, the amount of injected current and $\nabla$, the gradient operator. For complex conductivity distributions, analytical solution to the forward problem expressed in Eq.1 does not exist. Therefore, a numerical method must be applied. Finite element method (FEM) is used to calculate the electrical potential and corresponding magnetic flux density distribution for a given conductivity distribution and boundary condition.

After obtaining the electric potential distribution $U(r)$ from solving Eq.1, the electric field and the interior current density distribution are given as:

$$
\begin{aligned}
& E=-\nabla U \\
& J=\sigma E
\end{aligned}
$$

Then the magnetic flux density can be calculated using the Biot-Savat law: 


$$
B(r)=\frac{\mu_{0}}{4 \pi} \int_{\Omega} J\left(r^{\prime}\right) \times \frac{r-r^{\prime}}{\left|r-r^{\prime}\right|^{3}} d v^{\prime}
$$

where $B(r)$ is the magnetic flux density at the measurement point, $J(r)$ the current density at the source point and $\mu_{0}$ the magnetic permeability of the free space. In order to avoid the singularity occurring when $r=r, B(r)$ is treated as a node variable and $J(r)$ is used at the centre of each finite element in Eq.3. The comparison between the analytical solution and the numerical solution by FEM method was performed [20,21] to indicate the feasibility of solving the forward problem using the FEM.

\subsubsection{Inverse Problem}

For the inverse problem, the Radius Basic Function (RBF) Neural Network system was used to seek the optimal estimation eigenvalues of the target conductivities: $\lambda_{\text {long }}$ and $\lambda_{\text {trans }}$ of the WM, respectively. The conductivity of the gray matter $\sigma_{G M}$ and CSF $\sigma_{C S F}$ were assumed to be isotropic, thus each conductivity tensor has the same value in three directions. The eigenvector data of each element got from the DT-MRI measuring were combined with the initial sample eigenvalues belong to a certain range to perform the forward calculation. Based on the sample data $\boldsymbol{\delta}=\{\boldsymbol{B}, \boldsymbol{J}\}$ and the measured data " $\boldsymbol{\delta}$ " $=\left\{\boldsymbol{B}^{*}, \boldsymbol{J}^{*}\right\}$ " from the forward calculation, the objection function was set up and the RBF-MREIT algorithm was used to reconstruct the anisotropic conductivity eigenvalues of the WM.

In the RBF-MREIT algorithm description, the relationship between the estimated target values $\left[\lambda_{\text {long }}{ }^{\text {target }}\right.$, $\left.\lambda_{\text {trans }}^{\text {target }}\right]$ and the measured data " $\boldsymbol{\delta}^{*}=\left\{\boldsymbol{B}^{*}, \boldsymbol{J}^{*}\right\}$ " was considered as a "black box". The Radius Basic Function neural network was used herein to rebuild the inputoutput relation system. After the system was trained by the sample sets including the inputs and outputs couple data, the optimal parameters of the system were found through the optimization algorithm. Then the target values of the eigenvalues were reconstructed finally. There were two processes in the realization of the RBFMREIT algorithm: the function set up and the function optimization (See Figure 2).

\subsubsection{The Function Set Up}

The inputs of the function were assumed as the sample values of the unknown anisotropic eigenvalues $\left[\lambda_{\text {long }}, \lambda_{\text {trans }}\right]$ of the WM conductivity. The output was defined as the objective function $f\left(\sigma^{*}, \sigma\right)$ between the measured data " $\boldsymbol{\delta}^{*}=\left\{\boldsymbol{B}^{*}, \boldsymbol{J}^{*}\right\}$ " and the computed sample data $\boldsymbol{\delta}=\{\boldsymbol{B}, \boldsymbol{J}\}$.

$$
f\left(\sigma^{*}, \sigma\right)=\left(1-C C\left(\delta^{*}, \delta\right)\right)^{2}+R E\left(\delta^{*}, \delta\right)^{2}
$$

where $C C\left(\delta^{*}, \delta\right)$ and $\operatorname{RE}\left(\delta^{*}, \delta\right)$ denoted the correlation coefficient and relative error, respectively.

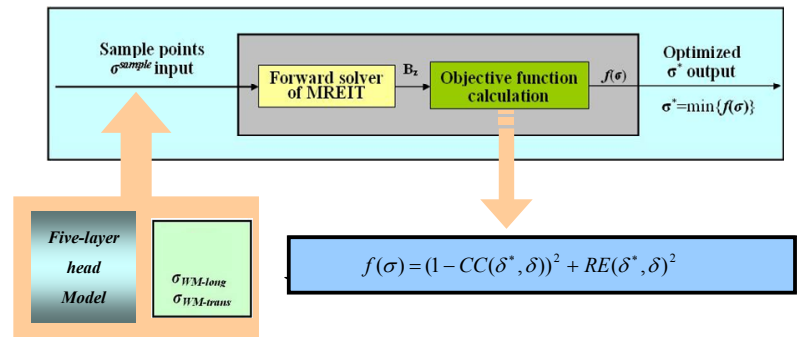

Figure 2. Flowchart of the RBF-MREIT algorithm frame.

\subsubsection{The Function Optimization}

Once the function between the input $\sigma$ and the output $f(\sigma)$ is obtained, optimization method, herein the simplex method, could be used to find the optimum point where the objective function $f(\sigma)$ is minimum and the measured data " $\boldsymbol{\delta}^{*}=\left\{\boldsymbol{B}^{*}, \boldsymbol{J}^{*}\right\}^{\prime}$ "were most close to the computed sample data set $\boldsymbol{\delta}=\{\boldsymbol{B}, \boldsymbol{J}\}$ as well as the target $\sigma^{*}$ to the estimated $\sigma$.

In conclusion, the inverse problem of the present RBF-MREIT algorithm could be realized as follows: Step 1: According to certain rules, some sampling input points $\sigma$ were chosen in the region of interest, and the corresponding objective functions $f(\sigma)$ were calculated by solving the forward problem. Step 2: The sampling input-output pairs were used to train the RBF network and the trained RBF network function $f(\sigma)$ was obtained. Step 3: Estimating the optimum input parameter $\sigma^{i}$ that minimizes the output value using the simplex method. Step 4: Resetting the region of interest by shrinking the old region of interest to a new region and choosing the optimum input parameters as the centre of the new region. Step 5: If the new region of interest was small enough or $\left\|\sigma^{i+1}-\sigma^{i}\right\| \leq \varepsilon$, then stop, otherwise, go to step 1, where $\sigma^{i+1}$ and $\sigma^{i}$ were the estimated conductivity distribution at the $(i+1)$ th and $i$ th iterations, respectively, and $\varepsilon$ was the allowable error.

\section{SIMULATIOIN STUDY}

In order to test the performance of RBF-MREIT algorithm, numerical simulations were performed on a concentric five-layer human head model (consisting of the scalp, skull, CSF, gray matter and WM) to estimate the unknown anisotropic conductivity eigenvalues of the WM. The results of the RBF-MREIT algorithm based on different data $\boldsymbol{\delta}$ were compared and analyzed.

\subsection{Simulation Parameters}

$5 \mathrm{~mA}$ bipolarity square-wave electric currents of $20 \mathrm{~Hz}$ frequency were injected into the head along the equator of the head model as shown in Figure 3, which allowed more signals flowing into the inner brain [20]. 7 electrode pair positions were chosen to inject the current in 


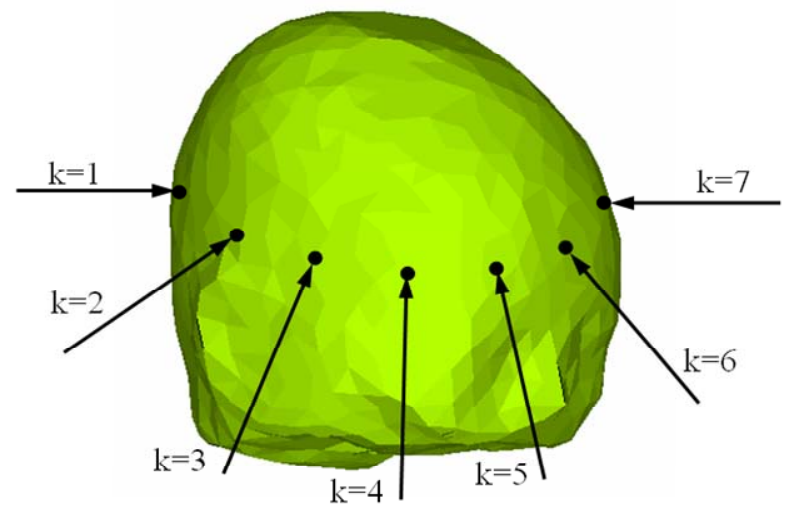

Figure 3. The locations of the electrodes and injected currents for MREIT on the five-layer FEM head model.

order to assess the effects of the injected current numbers to the imaging results under different SNR levels, MRI system was used to measure the magnetic flux density induced by the injected current, and further the current density distribution could be computed according to the electromagnetic theory. In our research, the measured data " $\boldsymbol{\delta}^{*}=\left\{\boldsymbol{B}^{*}, \boldsymbol{J}^{*}\right\}$ " were all simulated according to the given target conductivity distribution through the forward problem.

To test the noise tolerance of the algorithm, noises of different levels were added to the "measured" $\boldsymbol{B}_{z}$. The standard deviation of the added noise $\mathrm{S}_{B}$ was set [27] as:

$$
\begin{gathered}
\mathrm{S}_{B}=\frac{1}{2 \gamma T_{c} \mathrm{SNR}} \\
\mathrm{S}_{J}=\frac{1}{2 \gamma \mu_{0} T_{c} \mathrm{SNR}} \sqrt{\left(\frac{1}{\Delta x}\right)^{2}+\left(\frac{1}{\Delta y}\right)^{2}}
\end{gathered}
$$

where $\gamma=26.75 \times 10^{7} \mathrm{radT}^{-1} \mathrm{~s}^{-1}$ was the gyromagnetic ratio of hydrogen, $T_{c}$ the duration of injection current pulse of $50 \mathrm{~ms}$ and SNR the signal-to-noise ratio of the MR magnitude image. The SNR of the MR magnitude image with additive Gaussian White Noise (GWN) was set to be infinite, $90,60,30$ and 15 , respectively. $\Delta x$ and $\Delta y$ denoted the border of the element.

In this study, the relative error $(R E)$ between the estimated and the target conductivity distributions was used to quantitatively assess the performance of the MREIT reconstruction algorithm. The $R E$ was defined as:

$$
R E\left(\sigma, \sigma^{*}\right)=\frac{\left\|\sigma^{*}-\sigma\right\|}{\left\|\sigma^{*}\right\|} \times 100 \%
$$

where $\sigma^{*}$ was the target conductivity distribution and $\sigma$ the estimated conductivity distribution. Given the premised data and the known isotropic tissue conductivities of the scalp $\sigma_{\text {scalp }}=0.33 \mathrm{~s} / \mathrm{m}$, the skull $\sigma_{\text {skull }}=0.0165 \mathrm{~s} / \mathrm{m}$, the
CSF $\sigma_{C S F}=1.75 \mathrm{~s} / \mathrm{m}$ and the gray matter $\sigma_{G M}=1.75 \mathrm{~s} / \mathrm{m}[9$, 25], the unknown eigenvalues of the WM conductivities were reconstructed with the proposed RBF-MREIT algorithm based on different data $\delta$ (See Figure 4).

The target eigenvalues $\left[\lambda_{\text {long }}{ }_{\text {target }}=0.6498, \lambda_{\text {trans }}\right.$ target $=$ 0.06498] (See Table 1) for all the WM tissue elements of the FEM realistic head model were set according to [2, 6]. Sample values were selected in the domain: $\left[\lambda_{\text {long }}^{W M}\right.$, $\left.\lambda_{\text {trans }}^{W M}\right] \in(0,10) \times(0,1)$ as the eigenvalue of the WM tissue element. Then the "measured" $\boldsymbol{B}^{*}=\left[\boldsymbol{B}_{x}{ }^{*}, \boldsymbol{B}_{y}{ }^{*}, \boldsymbol{B}_{z}{ }^{*}\right]$ and calculated $\boldsymbol{J}^{*}=\left[\boldsymbol{J}_{x}{ }^{*}, \boldsymbol{J}_{\boldsymbol{y}}{ }^{*}, \boldsymbol{J}_{z}{ }^{*}\right]$ were calculated through the forward problem computation. Finally, the objective function was set up to search for the optimum value to minimize the function.

\subsection{Results}

Given the parameters assumed above, the inverse problem was solved by the RBF-MREIT to search for the optimum conductivity values. The reconstructed results based on the one component magnetic flux density $\boldsymbol{B}_{z}$ under different SNR levels were listed in Table 1, where $\mathrm{k}=1, \ldots, 7$ denoted the number of the injected currents.

Based on the data $\boldsymbol{\delta}=\left[\boldsymbol{B}_{z}\right]$ with five noise levels, the $R E \mathrm{~s}$ of the two estimated variables were less than $11 \%$. When SNR=15, the RE of the estimated eigenvalues of the two directions was about $10 \%$. In ideal situation without noise, the $R E$ was about $7 \%$. Table 1 showed that the present algorithm could reconstruct the conductivity distributions well even with the increase of the noise levels. The present simulation results demonstrated that the RBF-MREIT algorithm could reconstruct well the eigenvalues of the anisotropic conductivity image and was robust to the measurement noise.

Figure 5 showed the section of the target and reconstructed anisotropic WM conductivities, which gave more direct description. Figure 6 further illustrated the reconstruction results with various SNR when the number $\mathrm{k}$ of the current injected changed from 1 to 7 . From the results, we can see that the $R E \mathrm{~s}$ do not decrease when the number of the current injected increasing.

Figure 6 displayed that the accuracy was not propor-

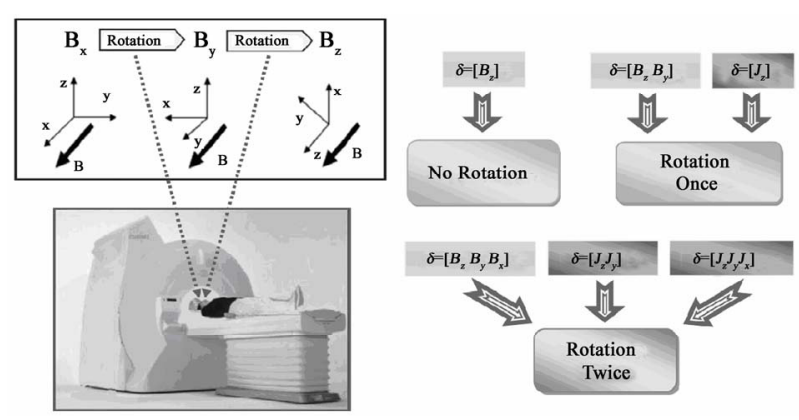

Figure 4. The composing of the objective function set up data $\delta$. 


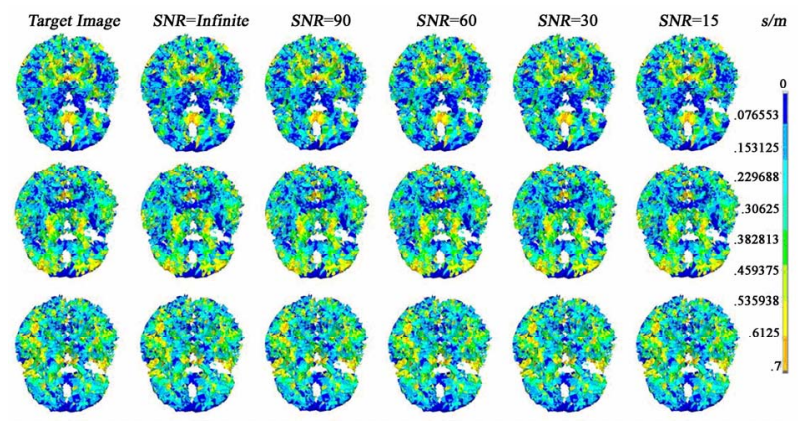

Figure 5. The target and the reconstructed conductivity image with different noise levels in three directions $\left(\boldsymbol{\delta}=\left[\boldsymbol{B}_{z}\right], \mathrm{k}=1\right)$.

tional to the numbers of the injected current for the reconstructed anisotropic conductivity image based on the RBF-MREIT algorithm. The same results could be got as well as the data $\boldsymbol{\delta}=\left[\boldsymbol{B}_{z} \boldsymbol{B}_{y}\right], \boldsymbol{\delta}=\left[\boldsymbol{B}_{z} \boldsymbol{B}_{\boldsymbol{y}} \boldsymbol{B}_{x}\right], \boldsymbol{\delta}=\left[\boldsymbol{J}_{z}\right], \boldsymbol{\delta}$ $=\left[\begin{array}{lll}\boldsymbol{J}_{z} & \boldsymbol{J}_{\boldsymbol{y}}\end{array}\right]$ and $\boldsymbol{\delta}=\left[\begin{array}{lll}\boldsymbol{J}_{z} & \boldsymbol{J}_{\boldsymbol{y}} & \boldsymbol{J}_{\boldsymbol{x}}\end{array}\right]$ were used to reconstruct the unknown eigenvalues of the WM tissue.

Figure 7 depicted the $R E$ s between the estimated and target conductivity distributions through different data $\delta$ $=\{\boldsymbol{B}, \boldsymbol{J}\}$ with different noise levels. Figure 7 indicated that the $R E \mathrm{~s}$ of the reconstructed results based on $\boldsymbol{\delta}=\left[\boldsymbol{B}_{z}\right]$ and $\boldsymbol{\delta}=\left[\boldsymbol{J}_{z}\right]$ are basically the same, as well as in the cases $\boldsymbol{\delta}$

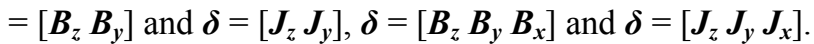
The reconstruction method using $\boldsymbol{\delta}=\left[\boldsymbol{B}_{z} \boldsymbol{B}_{y}\right], \boldsymbol{\delta}=\left[\begin{array}{ll}\boldsymbol{J}_{z} & \boldsymbol{J}_{\boldsymbol{y}}\end{array}\right]$, $\boldsymbol{\delta}=\left[\begin{array}{lll}\boldsymbol{B}_{z} & \boldsymbol{B}_{y} & \boldsymbol{B}_{x}\end{array}\right]$ and $\boldsymbol{\delta}=\left[\begin{array}{lll}\boldsymbol{J}_{z} & \boldsymbol{J}_{y} & \boldsymbol{J}_{\boldsymbol{x}}\end{array}\right]$ needed to rotate the human head at least once to twice in order to acquire the magnetic flux density and current density data. Especially, the method based on $\delta=\left[\begin{array}{llll}\boldsymbol{J}_{z} & \boldsymbol{J}_{\boldsymbol{y}} & \boldsymbol{J}_{\boldsymbol{x}}\end{array}\right]$ required the rotation which was not impractical in the clinic experiment.

Table 1. Reconstructed results of the WM for the realistic head model $\left(\boldsymbol{\delta}=\left[\boldsymbol{B}_{z}\right]\right)$

\begin{tabular}{|c|c|c|c|c|c|}
\hline \multirow{2}{*}{ SNR } & \multirow{2}{*}{ Current Inject } & \multicolumn{2}{|c|}{$\lambda_{1}^{\text {target }}=0.64890$} & \multicolumn{2}{|c|}{$\lambda_{t}^{\text {target }}=0.06489$} \\
\hline & & $\lambda_{1}$ & $R E$ & $\lambda_{\mathrm{t}}$ & $R E$ \\
\hline \multirow{7}{*}{ Infinite } & $\mathrm{k}=1$ & $0.6479 \pm 0.0112$ & $0.0790 \pm 0.0181$ & $0.0649 \pm 0.0012$ & $0.0760 \pm 0.0177$ \\
\hline & $\mathrm{k}=2$ & $0.6413 \pm 0.0025$ & $0.0691 \pm 0.0073$ & $0.0647 \pm 0.0002$ & $0.0659 \pm 0.0010$ \\
\hline & $\mathrm{k}=3$ & $0.6581 \pm 0.0040$ & $0.0608 \pm 0.0047$ & $0.0638 \pm 0.0004$ & $0.0688 \pm 0.0181$ \\
\hline & $\mathrm{k}=4$ & $0.6328 \pm 0.0033$ & $0.0754 \pm 0.0012$ & $0.0652 \pm 0.0000$ & $0.0502 \pm 0.0002$ \\
\hline & $\mathrm{k}=5$ & $0.6443 \pm 0.0011$ & $0.0753 \pm 0.0066$ & $0.0649 \pm 0.0000$ & $0.0661 \pm 0.0069$ \\
\hline & $\mathrm{k}=6$ & $0.6582 \pm 0.0002$ & $0.0726 \pm 0.0028$ & $0.0647 \pm 0.0003$ & $0.0699 \pm 0.0067$ \\
\hline & $\mathrm{k}=7$ & $0.6426 \pm 0.0042$ & $0.0619 \pm 0.0105$ & $0.0647 \pm 0.0005$ & $0.0687 \pm 0.0078$ \\
\hline \multirow{7}{*}{90} & $\mathrm{k}=1$ & $0.6499 \pm 0.0133$ & $0.0792 \pm 0.0182$ & $0.0650 \pm 0.0013$ & $0.0774 \pm 0.0185$ \\
\hline & $\mathrm{k}=2$ & $0.6582 \pm 0.0077$ & $0.0835 \pm 0.0127$ & $0.0654 \pm 0.0004$ & $0.0660 \pm 0.0111$ \\
\hline & $\mathrm{k}=3$ & $0.6424 \pm 0.0070$ & $0.0615 \pm 0.0056$ & $0.0663 \pm 0.0016$ & $0.0772 \pm 0.0026$ \\
\hline & $\mathrm{k}=4$ & $0.6415 \pm 0.0105$ & $0.0797 \pm 0.0056$ & $0.0639 \pm 0.0001$ & $0.0688 \pm 0.0016$ \\
\hline & $\mathrm{k}=5$ & $0.6357 \pm 0.0037$ & $0.0763 \pm 0.0105$ & $0.0643 \pm 0.0002$ & $0.0745 \pm 0.0078$ \\
\hline & $\mathrm{k}=6$ & $0.6495 \pm 0.0019$ & $0.0831 \pm 0.0037$ & $0.0657 \pm 0.0010$ & $0.0712 \pm 0.0090$ \\
\hline & $\mathrm{k}=7$ & $0.6414 \pm 0.0083$ & $0.0665 \pm 0.0108$ & $0.0641 \pm 0.0012$ & $0.0738 \pm 0.0080$ \\
\hline \multirow{7}{*}{60} & $\mathrm{k}=1$ & $0.6469 \pm 0.0133$ & $0.0810 \pm 0.0183$ & $0.0648 \pm 0.0014$ & $0.0813 \pm 0.0186$ \\
\hline & $\mathrm{k}=2$ & $0.6426 \pm 0.0103$ & $0.0859 \pm 0.0157$ & $0.0654 \pm 0.0005$ & $0.0665 \pm 0.0126$ \\
\hline & $\mathrm{k}=3$ & $0.6491 \pm 0.0083$ & $0.0666 \pm 0.0122$ & $0.0632 \pm 0.0021$ & $0.0795 \pm 0.0120$ \\
\hline & $\mathrm{k}=4$ & $0.6492 \pm 0.0115$ & $0.0802 \pm 0.0080$ & $0.0650 \pm 0.0002$ & $0.0874 \pm 0.0018$ \\
\hline & $\mathrm{k}=5$ & $0.6545 \pm 0.0077$ & $0.0863 \pm 0.0136$ & $0.0657 \pm 0.0010$ & $0.0786 \pm 0.0153$ \\
\hline & $\mathrm{k}=6$ & $0.6324 \pm 0.0051$ & $0.0884 \pm 0.0060$ & $0.0654 \pm 0.0017$ & $0.0765 \pm 0.0135$ \\
\hline & $\mathrm{k}=7$ & $0.6348 \pm 0.0179$ & $0.0688 \pm 0.0109$ & $0.0645 \pm 0.0012$ & $0.0825 \pm 0.0111$ \\
\hline \multirow{7}{*}{30} & $\mathrm{k}=1$ & $0.6510 \pm 0.0151$ & $0.0853 \pm 0.0194$ & $0.0650 \pm 0.0015$ & $0.0820 \pm 0.0194$ \\
\hline & $\mathrm{k}=2$ & $0.6473 \pm 0.0140$ & $0.0876 \pm 0.0169$ & $0.0647 \pm 0.0006$ & $0.0712 \pm 0.0154$ \\
\hline & $\mathrm{k}=3$ & $0.6633 \pm 0.0166$ & $0.0672 \pm 0.0278$ & $0.0652 \pm 0.0025$ & $0.0897 \pm 0.0006$ \\
\hline & $\mathrm{k}=4$ & $0.6380 \pm 0.0164$ & $0.0973 \pm 0.0164$ & $0.0641 \pm 0.0005$ & $0.0878 \pm 0.0068$ \\
\hline & $\mathrm{k}=5$ & $0.6518 \pm 0.0140$ & $0.0878 \pm 0.0171$ & $0.0658 \pm 0.0011$ & $0.0836 \pm 0.0154$ \\
\hline & $\mathrm{k}=6$ & $0.6445 \pm 0.0106$ & $0.1006 \pm 0.0061$ & $0.0636 \pm 0.0017$ & $0.0896 \pm 0.0372$ \\
\hline & $\mathrm{k}=7$ & $0.6446 \pm 0.0180$ & $0.0812 \pm 0.0112$ & $0.0664 \pm 0.0016$ & $0.0882 \pm 0.0190$ \\
\hline \multirow{7}{*}{15} & $\mathrm{k}=1$ & $0.6454 \pm 0.0155$ & $0.0855 \pm 0.0212$ & $0.0651 \pm 0.0016$ & $0.08561 \pm 0.02057$ \\
\hline & $\mathrm{k}=2$ & $0.6208 \pm 0.0203$ & $0.1067 \pm 0.0244$ & $0.0624 \pm 0.0017$ & $0.09077 \pm 0.04168$ \\
\hline & $\mathrm{k}=3$ & $0.6390 \pm 0.0178$ & $0.1027 \pm 0.0311$ & $0.0652 \pm 0.0025$ & $0.09318 \pm 0.01034$ \\
\hline & $\mathrm{k}=4$ & $0.6521 \pm 0.0231$ & $0.1020 \pm 0.0174$ & $0.0652 \pm 0.0009$ & $0.09352 \pm 0.01016$ \\
\hline & $\mathrm{k}=5$ & $0.6523 \pm 0.0229$ & $0.0989 \pm 0.0310$ & $0.0657 \pm 0.0013$ & $0.08864 \pm 0.01785$ \\
\hline & $\mathrm{k}=6$ & $0.6383 \pm 0.0160$ & $0.1038 \pm 0.0531$ & $0.0642 \pm 0.0021$ & $0.10713 \pm 0.05581$ \\
\hline & $\mathrm{k}=7$ & $0.6502 \pm 0.0189$ & $0.0839 \pm 0.0257$ & $0.0640 \pm 0.0031$ & $0.09791 \pm 0.02531$ \\
\hline
\end{tabular}




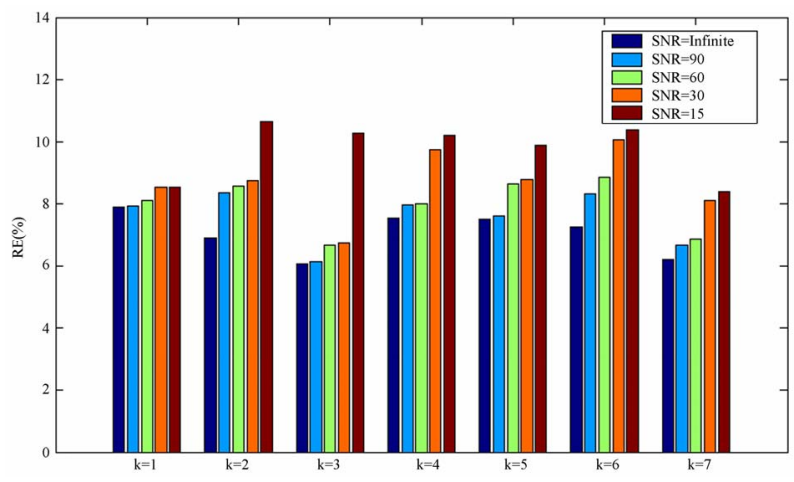

(a) $\lambda_{\text {long }}$

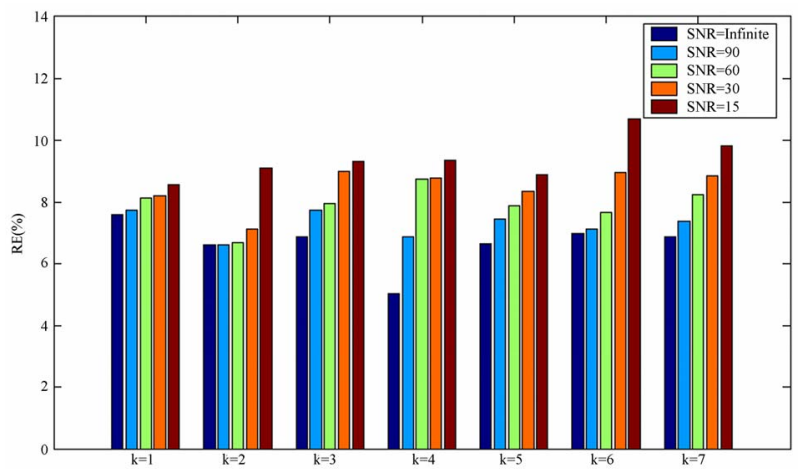

(b) $\lambda_{\text {trans }}$

Figure 6. The REs at different current injected numbers with different noise levels $\left(\boldsymbol{\delta}=\left[\boldsymbol{B}_{z}\right]\right)$.

Comparing the $R E$ s of results based on $\boldsymbol{\delta}=\{\boldsymbol{B}\}$ with five SNR noise levels, we can see that the $R E$ s using $\boldsymbol{\delta}=\left[\boldsymbol{B}_{z}\right]$ were relatively smaller than the other two results using $\delta$ $=\left[\boldsymbol{B}_{z} \boldsymbol{B}_{y}\right]$ and $\boldsymbol{\delta}=\left[\boldsymbol{B}_{z} \boldsymbol{B}_{y} \boldsymbol{B}_{x}\right]$. Even under the condition that number $\mathrm{k}$ of the injected currents changed from 1 to 7 , the same conclusion could be gained.

Figure 7 showed that the RBF-MREIT based on $\boldsymbol{\delta}=$ $\left[\boldsymbol{B}_{z}\right]$ could accurately detect the eigenvalues of the anisotropic WM conductivity in the deep brain region. This was a desired outcome of RBF-MREIT algorithm applied on the human head tissues. The RBF-MREIT algorithm based on $\boldsymbol{\delta}=\left[\boldsymbol{B}_{z}\right]$ without rotation was practical in further experimental study and provided a potential means for reconstructing the complex conductivities of the human brain and clinic application.

\section{DISCUSSION}

The RBF-MREIT algorithm, which was used to reconstruct the piece-wise homogeneous conductivity of three layer head model in [20], was extended to estimate the eigenvalues of the WM. Viewed from the microscopic angle, the conductivities of the human head are different everywhere and anisotropic characteristic exists everywhere, even for the same tissue. Some research $[6,28-30]$

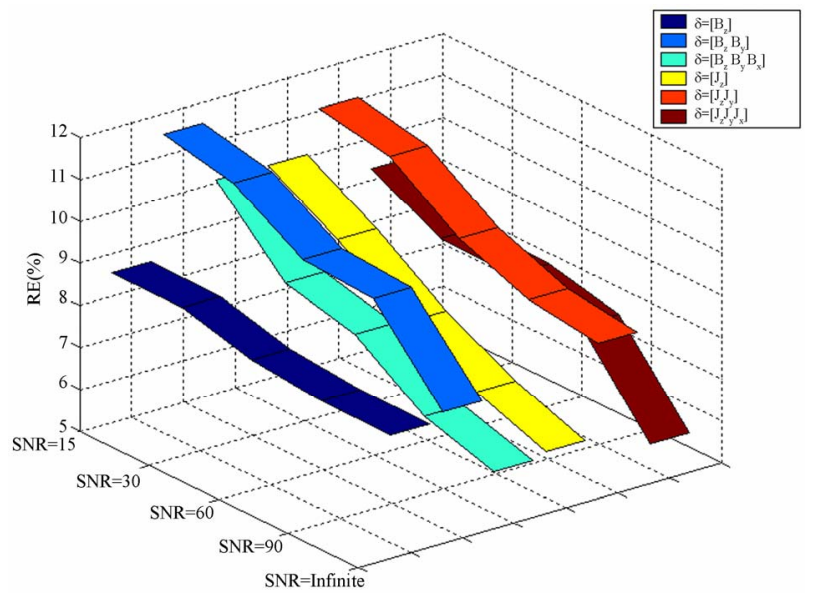

(a) $\lambda_{\text {long }}$

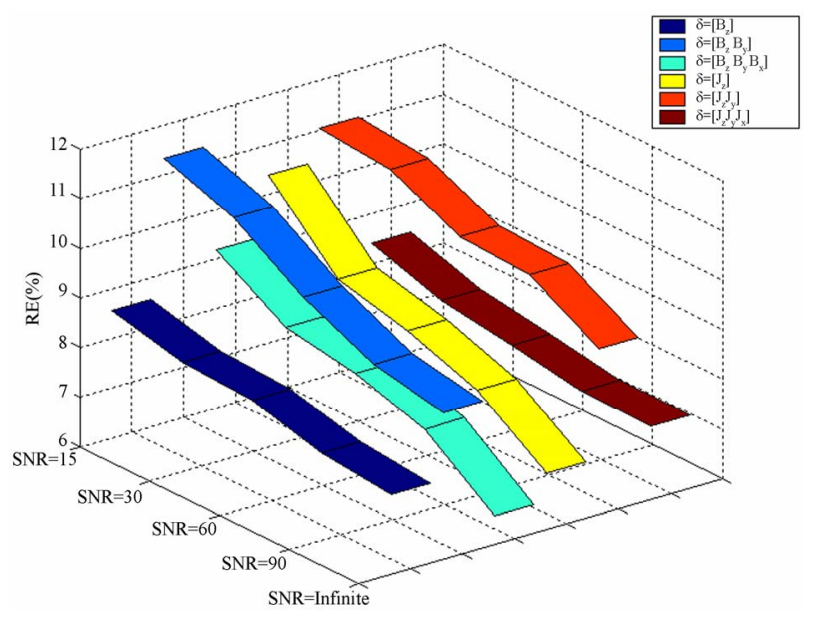

(b) $\lambda_{\text {trans }}$

Figure 7. The REs based on different data $\delta=\{\mathrm{B}, \mathrm{J}\}$ with different noise levels $(\mathrm{k}=1)$.

showed that the realistic head model with anisotropic and inhomogeneous conductivity could improve the accuracy of the source location in EEG/MEG analysis. Presently, the MREIT algorithms [20,21] were used in human head only under the condition of the piece-wise homogeneous conductivities of the tissues. Some other MREIT algorithms were not suitable to reconstruct the conductivities of the complex head tissues.

RBF-MREIT algorithm based on one component magnetic flux density solved the rotation problem in the MREIT research and had strong usability in the future clinic experiment. With different noise levels, all the $R E \mathrm{~s}$ of the reconstructed WM based on one component magnetic flux density $\boldsymbol{B}_{z}$ were less than $11 \%$, which was acceptable for the resolution requirement of the EEG/MEG analysis. The simulation results suggested that the algorithm was insensitive to the measurement noise. The present simulation results demonstrated the feasibility of the RBF-MREIT algorithm for anisotropic conductivity 
reconstruction of the human head tissues.

In summary, simulation results given above showed that the RBF-MREIT algorithm [20] extended to detect the eigenvalues of the anisotropic conductivity could reconstruct anisotropic WM conductivity distribution of the FEM human head model with high accuracy. The target anisotropic conductivity of the WM was set up based on the DT-MRI data according to the physical experiment measurement performed on a healthy human object. DT-MRI provides a noninvasive imaging modality to give more precise description of the human head tissues with anisotropic characteristic. So in our simulation, the head model considering the anisotropic tissues and based on the DT-MRI was reliable.

The target anisotropic conductivity defined in our research was based on Wolters' model [3,6], in which the eigenvalues of the anisotropic WM conductivity were assumed to be homogeneous. The model set up by Tuch $[2,5]$ was more complicated than Wolters', the eigenvalues of the anisotropic conductivity at every element being not identical. Wang [7] proposed a multi-compartment anisotropic WM model incorporating the partial volume effects of the CSF and the intra-voxel fiber crossing structure, which gave more refined description through the physiology angle. All these models could be the target values of the anisotropic conductivity reconstruction for our next work.

The RBF-MREIT algorithm has certain limitations on the application for the reconstruction of the inhomogeneous conductivities, especially on the complex human head tissues. More unknown values will increase the complexity and extend the training time of the Radius Basic Function Neural Network for the inhomogeneous conductivity reconstruction. All these will have impacts on the accuracy and efficiency of the RBF-MREIT algorithm. So the new MREIT algorithm, which is applied to reconstruct the inhomogeneous conductivity including the anisotropic conductivities of the human head tissue, is our future research focus.

In the simulation, a current of $5 \mathrm{~mA}$ was used, which is thought to be the upper safe limit for human beings (IEC criterion). And for human head, it is a little higher. So it would be desirable to utilize a better MRI scanner, some denoising techniques and improved methods. The information of the electrical properties of head tissues is used for electroencephalographic source localization and functional mapping of brain activities and functions. It has been proved that the information about vivo tissue conductivity values impacts the solution accuracy of bioelectrical field problems [28-30]. In our future studies, researches will focus on the study of continuous conductivity distribution reconstruction of the head tissues and the experimental validation of the algorithm on the hu- man head phantom experiment, as well as ways to reduce the amount of the injection current to less than $1 \mathrm{~mA}$ for human security consideration in clinical experiment.

\section{CONCLUSIONS}

We proposed the extended RBF-MREIT approach [20] based on one component measured magnetic flux density to avoid the rotation procedure for the noninvasive imaging of the three-dimensional conductivity distribution of the brain tissues with anisotropic characteristic. In this paper, the FEM method was used to build a five-layer head model constructed from DT-MRI data. Simulations were performed on the FEM model to reconstruct the conductivities of anisotropic tissues. A series of computer simulation results demonstrate the feasibility, the fast convergence ability and the improved robustness against measurement noise of the algorithm. Therefore, it is potential to provide a more accurate estimate of the WM anisotropic conductivity, and may have important applications to neuroscience research or clinical applications in neurology and neurophysiology.

However, the anisotropic conductivity estimation of the WM tissue is more useful to obtain high resolution source localization and mapping results. EEG/MEG inverse solution provides a unique tool to localize neural electrical activity of a human brain from noninvasive electromagnetic measurements. And it has been proved that information about the vivo tissue conductivity values improves the solution accuracy of bioelectrical field problems. Moreover, literatures [3, 6] show that the anisotropic conductivities of the skull and WM may have impact on EEG/MEG analysis and sensitivity of the source location in the Early Left Anterior Negativity (ELAN) usage of the language processing.

\section{REFERENCES}

[1] He, B. (2005) Neural engineering. Kluwer Academic Publishers, Norwell.

[2] Haueisen, J., Tuch, D.S., Ramon, C., Schimpf, P.H., Wedeen, V.J., George, J.S. and Belliveau, J.W. (2002) The influence of brain tissue anisotropy on human EEG and MEG. NeuroImage, 15(1), 159-166.

[3] Wolters, C.H., Anwander, A., Tricoche, X., Weinstein, D., Koch, M.A. and MacLeod, R.S. (2006) Influence of tissue conductivity anisotropy on EEG/MEG field and return current computation in a realistic head model: A simulation and visualization study using high-resolution finite element modeling. NeuroImage, 30(3), 813-826.

[4] Basser, P.J., Mattiello, J. and Lebihan, D. (1994) MR diffusion tensor spectroscopy and imaging. Biophysical Journal, 66(1), 259-267.

[5] Tuch, D.S., Wedeen, V.J., Dale, A.M., George, J.S. and Belliveau, J.W. (1999) Conductivity tensor mapping of 
the human brain using diffusion MRI. Annals of the New York Academy of Sciences, 888, 314-316.

[6] Wolters, C.H. (2002) Influence of tissue conductivity inhomogeneity and anisotropy on EEG/MEG based source localization in the human brain. Leipzig University, Leipzig.

[7] Wang, K., Zhu, S.A., Mueller, B., Lim, K., Liu, Z.M. and He, B. (2008) A new method to derive WM conductivity from diffusion tensor MRI. IEEE Transactions on Biomedical Engineering, 55(10), 2481-2486.

[8] Goncalves, S., de Munck, J.C., Heethaar, R.M. and da Silva, F.L. (2003) In vivo measurement of the brain and skull resistivities using an EIT-based method and realistic models for the head. IEEE Transactions on Biomedical Engineering, 50(6), 754-767.

[9] Lai, Y., van Drongelen, W., Ding, L., Hecox, K.E., Towle, V.L., Frim, D.M. and He, B. (2005) Estimation of in vivo human brain-to-skull conductivity ratio from simultaneous extra- and intra-cranial electrical potential recordings. Clinical Neurophysiology, 116(2), 456-465.

[10] Birgül, Ö., Eyüboğlu, B.M. and İder, Y.Z. (2003) Current constrainted voltage scaled reconstruction (CCSVR) algorithm for MR-EIT and its performance with different probing current patterns. Physics in Medicine and Biology, 48, 653-671.

[11] İder, Y.Z. and Birgül, Ö. (1998) Use of the magnetic field generated by the internal distribution of injected currents for electrical impedance tomography (MR-EIT). Elektrik, Turkish Journal of Electrical Engineering and Computer Sciences, 6(3), 215-225.

[12] Khang, H.S., Lee, B.I., Oh, S.H., Woo, E.J., Lee, S.Y., Cho, M.H., Kwon, O., Yoon, J.R. and Seo, J.K. (2002) $\mathrm{J}$-substitution algorithm in magnetic resonance electrical impedance tomography (MREIT): Phantom experiments for static resistivity images. IEEE Transactions on Medical Imaging, 21(6), 695-702.

[13] Kwon, O., Lee, J.Y. and Yoo, J.R. (2002) Equipotential line method for magnetic resonance electrical impedance tomography (MREIT). Inverse Problems, 18(2), 1089-1100.

[14] Özdemir, M.S., Eyüboğlu, B.M. and Özbek, O. (2004) Equipotential projection-based magnetic resonance electrical impedance tomography and experimental realization. Physics in Medicine and Biology, 49(20), 4765-4783.

[15] Seo, J.K., Yoon, J.R., Woo, E.J. and Kwon, O. (2003) Reconstruction of conductivity and current density imaging using only one component of magnetic field measurements. IEEE Transactions on Biomedical Engineering, 50(9), 1121-1124.

[16] Oh, S.H., Lee, B.I., Woo, E.J., Lee, S.Y., Cho, M.H., Kwon, O. and Seo, J.K. (2003) Conductivity and current density image reconstruction using harmonic $\mathrm{Bz}$ algorithm in magnetic resonance electrical impedance tomography. Physics in Medicine and Biology, 48(19), 31013116.

[17] Oh, S.H., Lee, B.I., Woo, E.J., Lee, S.Y., Kim, T.S., Kwon, O. and Seo, J.K. (2005) Electrical conductivity images of biological tissue phantom in MREIT. Physi- ological Measurement, 26(2), S279-S288.

[18] İder, Y.Z. and Onart, S. (2004) Algebric reconstruction for 3D magnetic resonance-electrical impedance tomography (MREIT) using one component of magnetic flux density. Physiological Measurement, 25(1), 281-294.

[19] Park, C., Kwon, O., Woo, E.J. and Seo, J.K. (2004) Electrical conductivity imaging using gradient $\mathrm{Bz}$ decomposition algorithm in magnetic resonance electrical impedance tomography (MREIT). IEEE Transactions on Medical Imaging, 23(3), 388-394.

[20] Gao, N., Zhu, S.A. and He, B. (2005) Estimation of electrical conductivity distribution within the human head from magnetic flux density measurement. Physics in Medicine and Biology, 50(11), 2675-2687.

[21] Gao, N., Zhu, S.A. and He, B. (2006) A new magnetic resonance electrical impedance tomography (MREIT) algorithm: the RSM-MREIT algorithm with applications to estimation of human head conductivity. Physics in Medicine and Biology, 51(12), 3067-3083.

[22] Gao, N. and He, B. (2008) Noninvasive imaging of bioimpedance distribution by means of current reconstruction magnetic resonance electrical impedance tomography. IEEE Transactions on Biomedical Engineering, 55(5), 1530-1539.

[23] Birgül, Ö. and İder, Y.Z. (1998) Use of magnetic field generated by the internal distribution of injected currents for electrical impedance tomography (MREIT). Elektrik, 6(3), 215-225.

[24] Seo, J.K., Pyo, H.C., Park, C., Kwon, O. and Woo, E.J. (2004) Image reconstruction of anisotropic conductivity tensor distribution in MREIT: Computer simulation study. Physics in Medicine and Biology, 49(18), 4371-4382.

[25] Zhang, Y.C., van Drongelen, W. and He, B. (2006) Estimation of in vivo brain-to-skull conductivity ratio in humans. Applied Physics Letters, 89(22), 2239032239033.

[26] Yao, Y., Zhu, S.A. and He, B. (2005) A method to derive FEM models based on BEM models. IEEE 27th Annual International Conference, Engineering in Medicine and Biology Society (EMBS), Shanghai, 1-4 September 2005, 1575-1577.

[27] Scott, G.C., Joy, M.L.G. and Armstrong, R.L. (1992) Sensitivity of magnetic-resonance current-density imaging. Journal of Magnetic Resonance, 97(2), 235-254.

[28] Awada, K.A., Jackson, D.R., Baumann, S.B., Williams, J.T., Wilton, D.R., Fink, P.W. and Prasky, B.R. (1998) Effect of conductivity uncertainties and modeling errors on EEG source localization using a 2-D model. IEEE Transactions on Biomedical Engineering, 45(9), 1135-1145.

[29] Ferree, T.C., Eriksen, K.J. and Tucker, D.M. (2000) Regional head tissue conductivity estimation for improved EEG analysis. IEEE Transactions on Biomedical Engineering, 47(12), 1584-1592.

[30] Gençer, N.G. and Acar, C.E. (2004) Sensitivity of EEG and MEG measurements to tissue conductivity. Physics in Medicine and Biology, 49(5), 707-717. 